\title{
Optimization for heat and sound insulation of honeycomb sandwich panel in thermal environments
}

\author{
Jinlong Yuan', Haibo Chen², Qiang Zhong ${ }^{3}$, Kongjuan $\mathrm{Li}^{4}$ \\ Department of Modern mechanics, University of Science and Technology of China, \\ CAS Key Laboratory of Mechanical Behavior and Design of Materials, Hefei, China \\ ${ }^{2}$ Corresponding author \\ E-mail: ${ }^{1} y u a n j i n l @ m a i l . u s t c . e d u . c n,{ }^{2} h b c h e n @ u s t c . e d u . c n,{ }^{3}$ qzhong@mail.ustc.edu.cn, \\ ${ }^{4}$ kongjli@mail.ustc.edu.cn
}

Received 13 April 2017; accepted 14 April 2017

DOI https://doi.org/10.21595/vp.2017.18481

Check for updates

\begin{abstract}
An optimization based on sequential quadratic programming (SQP) algorithm to increase the thermal insulation and sound transmission loss of honeycomb panel in thermal environments is presented. First, heat transfer analysis is performed to reveal the steady-state thermal performance of hexagonal aluminum honeycomb sandwich panel, by using the semi-empirical Swann and Pittman formula. Next, the influences of temperature on acoustic performance of honeycomb panel based on statistical energy analysis method (SEA) is performed. Results show that increasing the thickness of the honeycomb core can improve the acoustic performance and heat insulation behavior.
\end{abstract}

Keywords: honeycomb, heat insulation, sound transmission loss, SEA, optimization.

\section{Introduction}

The metal honeycomb sandwich panel is made up of upper and lower skin and intermediate honeycomb core, which is an important part of the metallic thermal protection system (MTPS) because of its relatively high rigidity, low structure density and certain thermal insulation performance [1]. In addition to the thermal protection structure, it is also widely used in other spacecraft bearing structure and the basic structure of large accessories, such as the sun wing of the substrate, aircraft engine parts [2]. When the aircraft is flying at high speed, the outer surface of the metal honeycomb panel will withstand a large aerodynamic thermal load and pneumatic noise, so its acoustic performance and heat insulation performance should meet certain requirements.

Vibro-acoustic studies of sandwich panels have been carried out with wave impedance analysis $[3,4]$ or statistical energy analysis $[5,6]$ in high frequency, but few of these studies considered thermal effects. Therefore, it is necessary to consider the thermal effect when studying acoustic performance of honeycomb panels.

This article is organized as follows: In Section 2, the numerical study is performed to reveal the influences of the cell size on the steady-state thermal performance of aluminum honeycomb sandwich panel, by using the semi-empirical Swann and Pittman formula. In Section 3, acoustic behavior of honeycomb panels is studied by considering the effect of temperature variation. In Section 4, the multi-optimization with the objective that simultaneously minimizing the sound transmission and the structural thermal transmission with constraint of area mass density is carried out here.

\section{Heat transfer analysis}

The heat transfer inside the honeycomb sandwich panel is very complex, which is the coupling process of solid, gas heat conduction, radiant heat transfer in the honeycomb cavity and natural convective heat transfer, scholars have proposed a variety of calculation models for the equivalent calculation of the heat transfer performance of the sandwich structure. Among them, the semi-empirical formula model proposed by Swann and Pittman [7] has been used as a standard model to calculate the equivalent thermal conductivity of the honeycomb structure, which has 
greatly facilitated the calculation of the heat transfer performance of the sandwich panel.

\subsection{Heat transfer calculation method}

In this paper, Swann and Pittman's semi-empirical formula is used to analyze the thermal performance of aluminum honeycomb sandwich panels. Swann and Pittman ignore the temperature gradient of the skin and treat the heat transfer in the thickness direction of the sandwich panel as a one-dimensional linear problem. The equivalent thermal conductivity of honeycomb sandwich considering solid, gas heat conduction and radiant heat transfer is expressed as:

$k_{e}=k_{f}\left(\frac{\Delta A}{A}\right)+k_{g}\left[1-\left(\frac{\Delta A}{A}\right)\right]+k_{r}$,

where $k_{f}$ and $k_{g}$ represent thermal conductivity of core and air, $\Delta A / A$ is the ratio of cell wall fraction to total cell area of the cross-sectional area of the honeycomb sandwich cell, $k_{r}$ radiation equivalent thermal conductivity, the expression is:

$k_{r}=0.664(\lambda+0.3)^{-0.69} \varepsilon^{1.63(\lambda+1)^{-0.89}} h\left(T_{1}+T_{2}\right)\left(T_{1}{ }^{2}+T_{2}{ }^{2}\right)$,

where $\lambda$ is the ratio of the core height to the straight hexagonal circumscribed circle diameter, $\varepsilon$ and $\sigma$ are surface panel emissivity and Stefan-Boltzmann constant, $T_{1}$ and $T_{2}$ are the temperature of the upper and lower panels. The equivalent thermal conductivity is related to the temperature of the upper and lower panels, therefore, the temperature of the lower panel can be calculated by repeated iterations.

\subsection{Heat insulation numerical calculation}

The size of the aluminum alloy honeycomb panels studied is as follows: the thickness of the upper and lower panels is $1 \mathrm{~mm}$, the height of the honeycomb core is $20 \mathrm{~mm}$, the wall thickness of the honeycomb core is $0.1 \mathrm{~mm}$, and the length of the honeycomb unit is $5 \mathrm{~mm}$. The emissivity of the honeycomb upper and lower plates is 0.25 . The thermal physical properties of aluminum alloy are shown in Table 1 . When the upper panel is subjected to a temperature of $300{ }^{\circ} \mathrm{C}$, the size effect of thermal insulation can be shown in Figs. 1-4.

Table 1. The thermal physical property parameter of aluminum alloy

\begin{tabular}{|c|c|c|c|c|}
\hline Temperature $/{ }^{\circ} \mathrm{C}$ & 25 & 100 & 200 & 300 \\
\hline Specific heat $/ \mathrm{J} /(\mathrm{kg} \cdot \mathrm{K})$ & 720 & 840 & 924 & 966 \\
\hline Thermal conductivity $/ \mathrm{W} /(\mathrm{m} \cdot \mathrm{K})$ & 147 & 151 & 160 & 168 \\
\hline
\end{tabular}

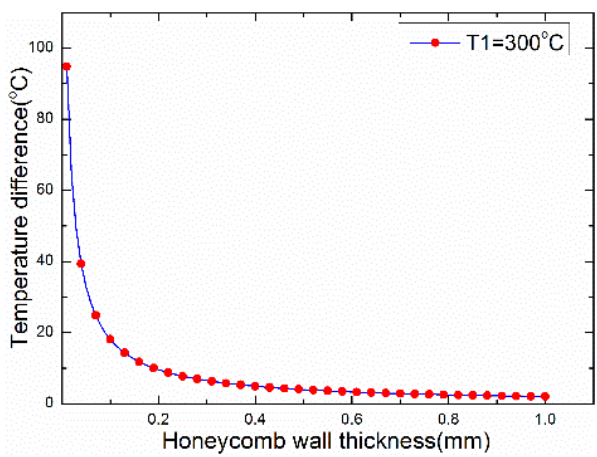

Fig. 1. The relationship between temperature difference and honeycomb wall thickness

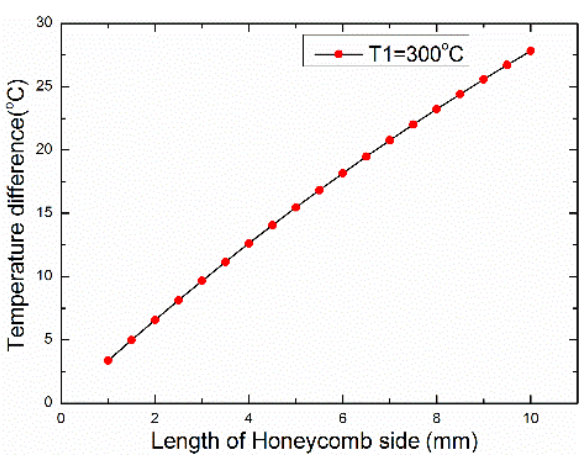

Fig. 2. The relationship between temperature difference and length of honeycomb side 


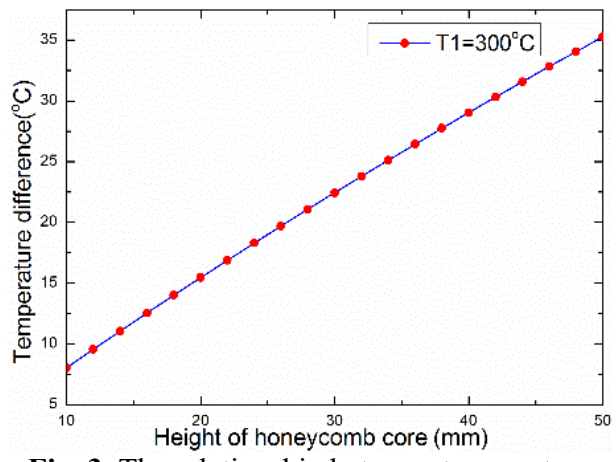

Fig. 3. The relationship between temperature

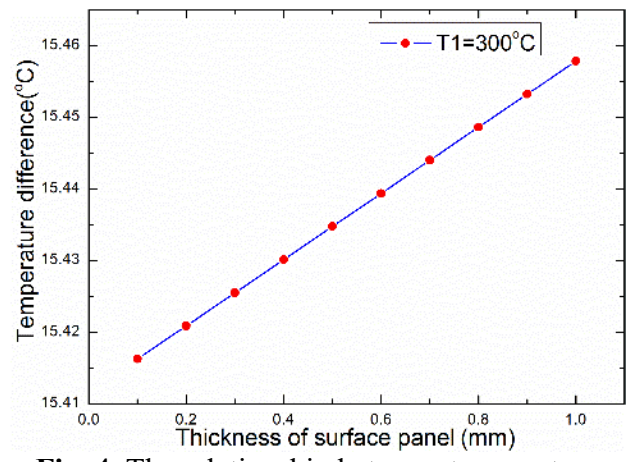

Fig. 4. The relationship between temperature difference and thickness of panel

The thermal conductivity of air is much smaller than the thermal conductivity of aluminum alloy, the larger the volume ratio of the aluminum alloy structure in the honeycomb core layer, the better the heat transfer. Therefore, the larger the honeycomb wall thickness, the smaller the temperature difference; the temperature difference increases with the honeycomb side length, panel thickness and honeycomb core thickness, as illustrated in Figs. 2-4.

\section{Acoustic performance}

Statistical energy analysis (SEA) is a modeling procedure which uses energy flow relationships for the theoretical estimation of the vibration response levels of structures in resonant motion.

\subsection{SEA theory}

The modal density of structures depends on their boundary conditions and the governing equations of motion. For simply supported panels, the modal density is associated with the constant frequency loci of the wavenumber; then:

$n(f)=2 \pi n(\omega)=\frac{A_{p}}{2} \frac{d k^{2}}{d \omega}$

where $A_{p}$ is the surface of panel.

For honeycomb sandwich panels with stiff cores, the anti-symmetric motion is dominant in the frequency under consideration. The sixth-order governing equation for free motion of sandwich structures presented by Mead and Markus [8] can be written as:

$k^{6}+g(1+Y) k^{4}-\frac{\mu}{D_{t}} \omega^{2} k^{2}-\frac{\mu}{D_{t}} g \omega^{2}=0$,

with:

$Y=\frac{\left[h+\left(t_{1}+t_{3}\right) / 2\right]^{2} E_{1} t_{1} E_{3} t_{3}}{D_{t}\left(E_{1} t_{1}+E_{3} t_{3}\right)}, \quad g=\frac{G_{c}}{h}\left(\frac{1}{E_{1} t_{1}}+\frac{1}{E_{3} t_{3}}\right), \quad D_{t}=\frac{E_{1} t_{1}^{3}+E_{3} t_{3}^{3}}{12}$,

where $E_{j}$ is the Young's modulus of the face sheet $j ; G_{c}$ the out-of-plane shear modulus of the core; $t_{j}$ and $h$ are the thickness of the face sheet $j$ and the core, and $\mu$ the mass area density of sandwich panel.

A transmission model is considered to consist of three coupled systems as source room, panel and receiving room, with the subscripts are expressed as 1,2 and 3. Hence, the transmission loss 
can be expressed as [6]:

$T L=10 \log _{10}\left[\frac{A_{p} T_{3}}{0.161 V_{3}}\left(\frac{E_{1} / V_{1}}{E_{3} / V_{3}}-1\right)\right]$,

with:

$\frac{E_{1} / n_{1}}{E_{3} / n_{3}}-1=\frac{2 \eta_{\text {rad }} n_{2} \eta_{3} n_{3}+\left(\eta_{3} n_{3}+\eta_{\text {rad }} n_{2}\right) \eta_{2} n_{2}}{\eta_{\text {rad }}^{2} n_{2} n_{3}+\eta_{13}\left(2 \eta_{\text {rad }}+\eta_{2}\right) n_{1} n_{2}}$

where $V_{j}$ is $T(K)$ the volume of source room and receiving room, $\eta_{j}$ and $n_{j}$ are the internal loss factor and modal density. $\eta_{\text {rad }}$ is the coupling loss factor due to radiation damping, $R_{\text {rad }}$ is the radiation resistance of a baffled simply supported single-layer panel given by [9]. The internal loss factor of the sound field was determined from the reverberation time $T_{3}$, the modal density can be obtained from Eqs. (1-2).

\subsection{Effect of temperature on acoustic performance}

When only considering the effect of temperature on the physical parameters, a typical temperature-dependent material properties $P$ can be expressed as follows [10]:

$Y=H_{0}\left(H_{-1} T^{-1}+1+H_{1} T+H_{2} T^{2}+H_{3} T^{3}\right)$,

where $H_{i}$ is the coefficients of temperature $T(K)$, which are particular to each specific material. Elastic modulus $E$ of aluminum alloy are taken as temperature dependent. The temperature-dependent coefficients are listed in Table 2.

Table 2. The thermal coefficient of elastic modulus E of aluminum alloy

\begin{tabular}{|c|c|c|c|c|}
\hline$H_{-1}(K)$ & $H_{0}(K)$ & $H_{1}(K)$ & $H_{2}(K)$ & $H_{3}(K)$ \\
\hline 0 & 204.2 & $-4.585 \mathrm{E}-3$ & $1.050 \mathrm{E}-5$ & $-8.338 \mathrm{E}-9$ \\
\hline
\end{tabular}

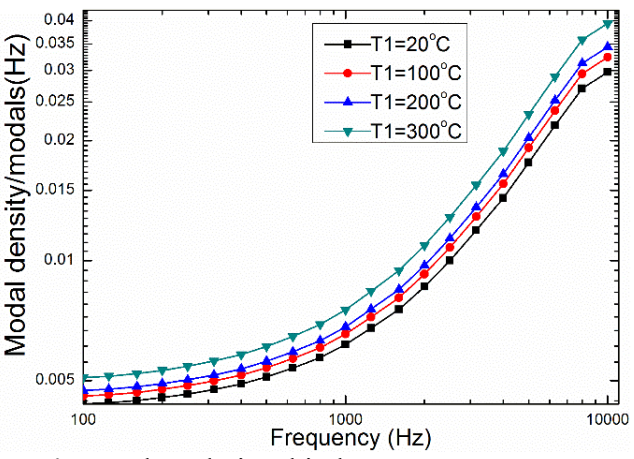

Fig. 5. The relationship between temperature and modal density of honeycomb sandwich panel

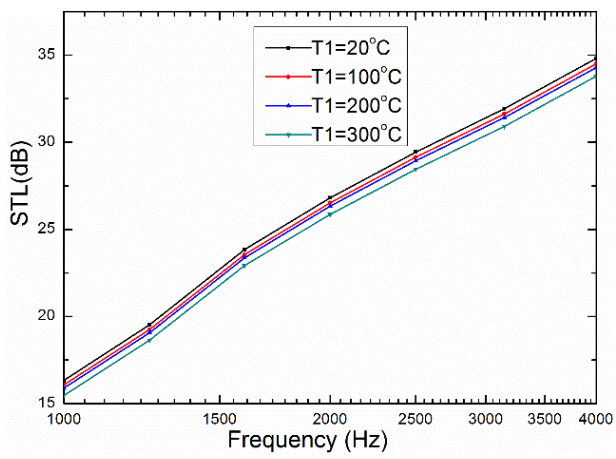

Fig. 6. The relationship between temperature and sound transmission loss of honeycomb sandwich panel

The dimensions of the panels are $0.84 \mathrm{~m} \times 0.42 \mathrm{~m} . T_{3}$ and $\eta_{2}$ are set as $1.4 \mathrm{~s}$ and 0.03 . Because the thickness of the upper and lower panels is very small, the temperature of upper and lower panels can be considered homogeneous. The temperature of honeycomb is assumed to be intermediate temperature of the core approximately. Modal density increases with temperature shown in Fig. 5, and increasing the temperature causes the sound transmission loss to decrease as illustrated in Fig. 6, the main reason is that the increase of temperature leads to the decrease of 
elastic modulus.

\section{Optimization analysis}

In this part, a multi-objective optimization model of honeycomb sandwich is proposed, with the objective of maximizing the sound transmission loss and thermal insulation. The thickness of the upper and lower panels, height of honeycomb core, the wall thickness of the honeycomb core and the length of the honeycomb unit are chosen as the decision variable.

\subsection{Objective function}

The acoustical function is determined from a weighted average of the field-incidence transmission coefficients:

$\bar{\tau}_{\text {ave }}=\sum_{i=1}^{7} w_{i} \tau_{i}$,

in which the weighting function is chosen to correspond to a weighting for seven frequencies in the range of $1000-4000 \mathrm{~Hz}$ [11]. Finally, the weighted sound transmission loss average was calculated from:

$A T L=-10 \lg \left|\bar{\tau}_{\text {ave }}\right|$,

thermal insulation object function was set as:

$\Delta T=T_{1}-T_{3}$.

In this paper, the constraint is the area mass density:

$\mu=\rho_{f}\left(t_{1}+t_{3}\right)+\rho_{c} t_{2}$,

where $\rho_{f}$ and $\rho_{c}$ are the density of face panel and core. Therefore, Multi-objective optimization problem can be written:

$\min \quad F=\frac{w_{1} A T L_{0}}{A T L}+\frac{w_{2} \Delta T_{0}}{\Delta T}$,

s.t. $\left\{\begin{array}{l}\mu \leq \mu_{\max }, \\ 0.1 \mathrm{~mm} \leq t_{f} \leq 1 \mathrm{~mm}, \\ 10 \mathrm{~mm} \leq t c \leq 50 \mathrm{~mm}, \\ 0.01 \mathrm{~mm} \leq t \leq 1 \mathrm{~mm}, \\ 1 \mathrm{~mm} \leq l \leq 10 \mathrm{~mm},\end{array}\right.$

where $w_{1}$ and $w_{2}$ denote the weight of objective function, satisfied with $w_{1}+w_{2}=1 . A T L_{0}$ and $\Delta T_{0}$ were single objective optimization result of $A T L$ and $\Delta T . \mu_{\max }=4 \mathrm{~kg} / \mathrm{m}^{2}$ is used in the following numerical calculation.

\subsection{Optimization}

The SQP algorithm is an efficient method to solve the nonlinear programming problem. The basic idea is to construct a simple approximation optimization problem by using the information about the nonlinear programming problem. The current iteration point is corrected by solving it, with a series of linear programming or quadratic programming to successively approximate the 
original nonlinear programming problem.

$A T L_{0}=20.4951 \mathrm{~dB}$ and $\Delta T_{0}=157.6101^{\circ} \mathrm{C}$ are obtained by SQP, with constraint of area mass density. When $w_{1}$ and $w_{2}$ are set as 0.5 , the iterative process of the multi-objective function value is shown in Fig. 7. The optimization results are achieved when the height of honeycomb core reaches a maximum, the relationship between ATL and $\Delta T$ is displayed in Fig. 8.

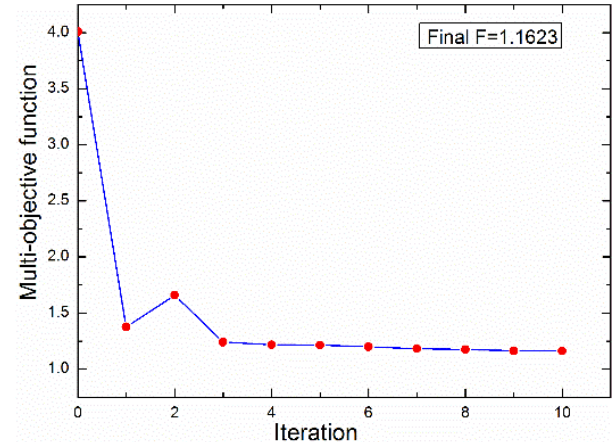

Fig. 7. The iterative process of the multi-objective function value

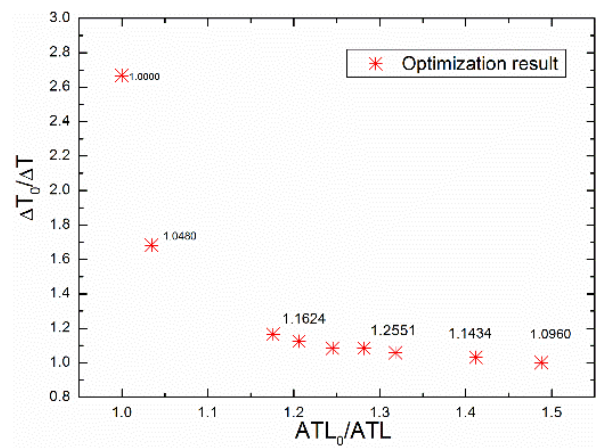

Fig. 8. The relationship between ATL and $\Delta T$

\section{Conclusions}

Aluminum alloy honeycomb insulation performance is related to size, the main factor is related to the proportion of aluminum material to the core. The increase of temperature leads to the decrease of elastic modulus and the increase of modal density, eventually leading to reduced sound transmission loss. Increasing the core thickness helps to improve the thermal insulation and sound insulation of the honeycomb panel at the same time. The optimized result has an important guidance value for engineering applications.

\section{References}

[1] Caogen Y., Hongjun L., Zhonghua J., et al. A study on metallic thermal protection system panel for reusable launch vehicle. Acta Astronautica, Vol. 63, Issue 1, 2008, p. 280-284.

[2] Shang G. Q., Zhu Z. S., Chang H., et al. Development of ultra-high strength titanium alloy. Chinese Journal of Rare Metals, Vol. 35, Issue 1, 2011, p. 286-291.

[3] Kurtze G., Watters B. G. New wall design for high transmission loss or high damping. The Journal of the Acoustical Society of America, Vol. 31, Issue 6, 1959, p. 739-748.

[4] Dym C. L., Lang M. A. Transmission of sound through sandwich panels. The Journal of the Acoustical Society of America, Vol. 56, Issue 5, 1974, p. 1523-1532.

[5] Wang T., Li S., Rajaram S, et al. Predicting the sound transmission loss of sandwich panels by statistical energy analysis approach. Journal of Vibration and Acoustics, Vol. 132, Issue 1, 2010, p. 256-280.

[6] Zhou R., Crocker M. J. Sound transmission loss of foam-filled honeycomb sandwich panels using statistical energy analysis and theoretical and measured dynamic properties. Journal of Sound and Vibration, Vol. 329, Issue 6, 2010, p. 673-686.

[7] Swann R. T., Pittman C. M. Analysis of Effective Thermal Conductivities of Honeycomb-Core and Corrugated-Core Sandwich Panels. National Aeronautics and Space Administration, 1961.

[8] Mead D. J., Markus S. The forced vibration of a three-layer, damped sandwich beam with arbitrary boundary conditions. Journal of Sound and Vibration, Vol. 10, Issue 2, 1969, p. 163-175.

[9] Crocker M. J., Price A. J. Sound transmission using statistical energy analysis. Journal of Sound and Vibration, Vol. 9, Issue 3, 1969, p. 469-486.

[10] Touloukian Y.S. Thermophysical Properties of High Temperature Solid Materials. Macmillan, New York, 1967.

[11] Makris S. E., Dym C. L., Smith J. M. G. Transmission loss optimization in acoustic sandwich panels. The Journal of the Acoustical Society of America, Vol. 79, Issue 6, 1986, p. 1833-1843. 